\title{
Mutational landscape of radiation-associated angiosarcoma of the breast
}

\author{
Bryan J. Thibodeau ${ }^{1}$, Vincent Lavergne ${ }^{2}$, Nayana Dekhne ${ }^{3}$, Pamela Benitez ${ }^{3}$, \\ Mitual Amin ${ }^{4}$, Samreen Ahmed ${ }^{1}$, Jean L. Nakamura ${ }^{2}$, Philip R. Davidson ${ }^{5}$, Alice 0. \\ Nakamura ${ }^{5}$, Inga S. Grills ${ }^{6}$, Peter Y. Chen ${ }^{6}$, Jessica Wobb ${ }^{6}$ and George D. Wilson ${ }^{1,6}$

Copyright: Thibodeau et al. This is an open-access article distributed under the terms of the Creative Commons Attribution License 3.0 (CC BY 3.0), which permits unrestricted use, distribution, and reproduction in any medium, provided the original author and source are credited.

\section{ABSTRACT}

Purpose: Radiation-associated breast angiosarcomas are a rare complication of radiation therapy for breast carcinoma. With relatively little is known about the genetic abnormalities present in these secondary tumors, we examined genomic variation in biospecimens from radiation-associated breast angiosarcomas.

Experimental Design: Patients were identified that had a previous breast cancer diagnosis, received radiation therapy, and developed angiosarcoma in the ipsilateral breast as the earlier cancer. Tumor regions were isolated from archival blocks using subsequent laser capture microdissection. Next generation sequencing was performed using a targeted panel of $\mathbf{1 6 0}$ cancer-related genes. Genomic variants were identified for mutation and trinucleotide-based mutational signature analysis.

Results: $\mathbf{4 4}$ variants in $\mathbf{3 4}$ genes were found in more than two thirds of the cases; this included 12 variants identified as potentially deleterious. Of particular note, the BRCA1 DNA damage response pathway was highly enriched with genetic variation. In a comparison to local recurrences, 14 variants in 11 genes were present in both the primary and recurrent lesions including variants in genes associated with the DNA damage response machinery. Furthermore, the mutational signature analysis shows that a previously defined IR signature is present in almost all of the current samples characterized by predominantly $\mathrm{C} \rightarrow$ T substitutions.

Conclusions: While radiation-associated breast angiosarcomas are relatively uncommon, their prognosis is very poor. These data demonstrate a mutational pattern associated with genes involved in DNA repair. While important in revealing the biology behind these tumors, it may also suggest new treatment strategies that will prove successful.

\section{INTRODUCTION}

Angiosarcomas are a relatively rare histological subtype of sarcomas and represent approximately $1 \%$ of all sarcomas [1]. They display remarkable clinical heterogeneity and can occur anywhere in the body, although the breast is a frequent location. Secondary sarcomas are a recognized complication of radiation therapy for breast carcinoma and are associated with poor prognosis [2]. The estimated incidence of treatmentassociated breast angiosarcoma is $0.002-0.05 \%$ per year corresponding to $\sim 40 \%$ of all radiation-associated 
sarcomas that develop after radiotherapy [3]. Radiationassociated breast angiosarcomas have poor prognosis and relatively little is known about the genetic abnormalities present in these secondary tumors. Recent evidence has implicated amplification of the $8 \mathrm{q} 24.2$ region containing the $M Y C$ gene in secondary but not in primary breast angiosarcomas [4]. High expression of the Myc protein was found to be associated with amplification, suggesting that MYC amplification may be implicated in the pathology of secondary breast angiosarcomas [5].

A recent study of 7 primary breast angiosarcomas and 18 secondary breast angiosarcomas arising in the irradiation field of a radiotherapy were analyzed for copy number alterations and differential gene expression using Affymetrix SNP 6.0 Array and Affymetrix Exon Arrays [6]. This study showed that two transcriptome signatures of the radiation tumorigenesis coexisted in these tumors. One was histology specific and correctly discriminated $100 \%$ of the primary tumors from the radiation-associated tumors. The deregulation of marker genes, including podoplanin (PDPN), prospero homeobox 1 (PROX1), vascular endothelial growth factor 3 (VEGFR3) and endothelin receptor A (EDNRA), suggests that the radiation-associated breast angiosarcomas developed from radiation-stimulated lymphatic endothelial cells. In addition, the authors showed that the high rate of MYC amplification found in radiation-associated breast angiosarcomas is likely a consequence of genome instability initiated by ionizing radiation; however, they suggest that it is not a marker of the radiation tumorigenesis since it was also observed at a low rate in primary tumors.

There is currently no data on specific mutations in radiation-associated breast angiosarcomas. In this study we used next generation DNA sequencing to investigate genomic variation specific to radiation-associated angiosarcomas. To assess the similarity of our radiationassociated breast angiosarcomas to other radiationinduced malignancies described in the literature [7-9], we performed non-negative matrix factorization (NMF) trinucleotide mutational signature analysis. In addition we used this variation to determine if particular signaling pathways were preferentially altered.

\section{RESULTS}

\section{Patient data}

Table 1 includes clinical and demographic data for 13 cases of radiation-associated angiosarcoma and 3 cases of sporadic angiosarcoma. Clinical data for the angiosarcoma as well as the initial breast cancer is included for patients with radiation-associated angiosarcoma. All but one of the radiation-associated angiosarcomas were treated by surgical mastectomy, and 9 of the 13 had grade 3 disease. While there were 4 local recurrences, only 1 went on to develop distant metastasis. Interestingly the earlier breast cancer tended to be stage I or II and estrogen receptor positive. The vast majority of the initial breast cancers were treated with whole breast irradiation with more than $60 \%$ also receiving hormone therapy. The median interval between the initial breast cancer and the radiation-associated angiosarcoma was 6.8 years with a range from 3.9 to 13.5 years.

\section{Radiation-associated angiosarcoma}

Next generation sequencing was performed on the Illumina NextSeq using a panel of 160 cancer-related genes. Variants were initially filtered to include only those found in more than two thirds ( $\geq 9$ of 13) of the cases of radiation-associated angiosarcoma. There were 44 variants in 34 different genes (Supplementary Table 2) including 12 variants that were identified as potentially deleterious (Table 2). Variants were recognized as potentially deleterious by meeting one of the following criteria: (i) categorized as pathogenic or likely pathogenic based upon ACMG guidelines, (ii) listed in HGMD/ClinVar, or (iii) having a CADD (Combined Annotation Dependent Depletion) score greater than 20. Among these variants, 3 missense mutations (EGFR c.1496G > A / p.Cys499Tyr; BRAF c.1915G > A / and c.1894C > T / p.Pro632Ser) were found in all 13 cases. The p.Val639Ile mutation in BRAF has been annotated in COSMIC as associated with squamous-cell carcinoma in the lung.

Further characterization of the variants present in the radiation-associated angiosarcomas was done with Ingenuity Variant Analysis to investigate signaling pathways preferentially affected in radiation-associated angiosarcomas. The "role of BRCA1 in DNA damage response" was highly enriched with genetic variation. In order to focus on variants shown to have a damaging effect, filtering was applied to include only those identified as: (i) pathogenic or likely pathogenic by $\mathrm{HCMG}$, (ii) damaging or activating by SIFT function prediction [10], or (iii) probably damaging, possible damaging, or damaging by PolyPhen 2 function prediction [11]. This demonstrated that all 13 cases had at least 2 genes affected in this pathway (Figure 1). In total, 17 different genes in the DNA damage response system contained a variant in one of the cases of radiation-associated angiosarcoma (Supplementary Table 3). Each patient sample had an average of 6 genes with a non-synonymous variant (range 2-11).

\section{Comparison to local recurrence}

In addition to the 13 radiation-associated angiosarcomas, two patients had tissue available from the resulting local recurrence (patient ID AX5613 and AX5616 in Table 1). Only 2 variants (the missense variant BUB1B c.2161C > A / p.Pro721Thr and the synonymous 
Table 1: Patient data

\begin{tabular}{|c|c|c|c|c|c|c|c|c|c|c|c|c|c|}
\hline \multirow{2}{*}{$\begin{array}{l}\text { Patient } \\
\text { ID }\end{array}$} & \multirow{2}{*}{$\begin{array}{l}\text { Angio } \\
\text { Type }\end{array}$} & \multirow{2}{*}{ age $d x$} & \multirow{2}{*}{$\begin{array}{c}\text { Angio Surgery } \\
\text { type }\end{array}$} & \multirow{2}{*}{ grade } & \multirow{2}{*}{ chemo } & \multirow{2}{*}{ LR } & \multirow{2}{*}{ DM } & \multirow{2}{*}{$\begin{array}{l}\text { Interval } \\
\text { (month) }\end{array}$} & \multicolumn{5}{|c|}{ Earlier Breast Cancer } \\
\hline & & & & & & & & & Stage & ER/PR/Her2 & chemo & hormones & XRT \\
\hline AX5612 & Rad-Ind & 74 & simple mastectomy & 3 & no & + & - & 109 & IA & $+/ \mathrm{ND} / \mathrm{ND}$ & no & novaldex & WBI \\
\hline AX5613 & Rad-Ind & 77 & simple mastectomy & 3 & no & + & - & 87 & IA & $+/ \mathrm{ND} /-$ & no & tamoxifen & WBI \\
\hline AX5617 & Rad-Ind & 86 & $\begin{array}{l}\text { incisional biopsies } \\
\text { only }\end{array}$ & 3 & no (refused) & - & - & 68 & IA & $98 \% / 2 \% / 0+$ & no & no & WBI \\
\hline AX5618 & Rad-Ind & 59 & mastectomy & 2 & $\begin{array}{l}\text { doxycycline, } \\
\text { ifosfamide, } \\
\text { mesnax } 4\end{array}$ & + & + & 162 & IA & $94 \%$ / ND / ND & no & tamoxifen & WBI \\
\hline AX5615 & Rad-Ind & 74 & simple mastectomy & 3 & no & - & - & 81 & IIA & $1 \% / 2 \% / \mathrm{ND}$ & no & no & WBI \\
\hline AX5626 & Rad-Ind & 75 & simple mastectomy & 3 & no & - & - & 105 & IA & $-/-/ \mathrm{ND}$ & no & tamoxifen & WBI \\
\hline AX5625 & Rad-Ind & 62 & simple mastectomy & 3 & $\begin{array}{c}\text { yes } \\
\text { (unknown) }\end{array}$ & - & - & 75 & IA & $99 \% / 12 \% / 0+$ & no & arimidex & WBI \\
\hline AX5622 & Rad-Ind & $* *$ & mastectomy & $* *$ & $* *$ & $* *$ & $* *$ & $* *$ & $* *$ & ** & $* *$ & raloxifene & ** \\
\hline AX5628 & Rad-Ind & 82 & simple mastectomy & 3 & no & - & - & 107 & IIA & $74 \% / \mathrm{ND} /-$ & no & no & WBI \\
\hline AX5616 & Rad-Ind & 82 & $\begin{array}{l}\text { mastectomy/wide } \\
\text { local excision with } \\
\text { graft closure }\end{array}$ & 3 & no & + & - & 60 & IA & $91 \% / 16 \% / 0+$ & no & no & 3D-CRT \\
\hline AX5619 & Rad-Ind & 44 & simple mastectomy & 2 & $\begin{array}{c}\text { neoadjuvant } \\
\text { taxol }\end{array}$ & - & - & 75 & IIA & $67 \% / 73 \% /-$ & ACT & tamoxifen & WBI \\
\hline AX5614 & Rad-Ind & $* *$ & simple mastectomy & 2 & $\begin{array}{c}\text { neoadjuvant } \\
\text { taxol }\end{array}$ & - & - & $* *$ & $* *$ & $* *$ & $* *$ & $* *$ & $* *$ \\
\hline AX5621 & Rad-Ind & 88 & nodule excision & 3 & $\mathrm{TMZ}$ & - & - & 47 & IIIA & $100 \% / 87 \% / 0+$ & no & tamoxifen & WBI \\
\hline AX5609 & Sporadic & 39 & $* *$ & $* *$ & no & $* *$ & $* *$ & & & & & & \\
\hline AX5627 & Sporadic & 75 & partial resection & & no & - & - & & & & & & \\
\hline AX5630 & Sporadic & 70 & simple mastectomy & 3 & $* *$ & - & + & & & & & & \\
\hline
\end{tabular}

Angiosarcoma type: radiation-induced (Rad-Ind) and sporadic Local recurrence (LR): yes (+), no (-). Distant metastasis (DM): yes (+), no (-). Estrogen Receptor (ER)/ Progesterone Receptor (PR)/Her2: non-determined (ND). Radiation treatment (XRT): whole breast (WBI), 3D-CRT (3-dimensional conformal radiotherapy). ${ }^{* *}$ : not available/ unknown.

variant EP300 c.2871T > A / p.T957T) occurred in the primary angiosarcoma that did not occur in the local recurrence; however, 14 variants in 11 genes were present in both the primary and recurrent lesions (including 2 missense variants and 1 splice site loss, Table 3). This includes variants in 4 genes (ATM, BRCA1, BRCA2, MSH6) associated with the DNA damage response machinery. Also of interest may be the 14 variants (including 7 non-synonymous variants) in 11 genes that are present in the local recurrence and not in the primary angiosarcoma (Supplementary Table 4). Among these are 6 variants that are predicted to be pathogenic / damaging by the IVA software, including variants in JAK1 (c.3047T $>$ A, / p.Val1016Glu), TERT (c.1883A > C / p.Asp628Ala), KMT2D (c.1185G > T / p.Gln395His), ERCC5 (c.2431A > G / p.Ser1265Gly), DICER1 (c.3572T > A / p.Leu1191*), and EP300 (c.6881T > A / p.Leu2294Gln).

\section{Specific to sporadic angiosarcoma}

In order to better identify variants that may be specific to radiation-associated angiosarcoma, 3 cases of angiosarcoma that developed in the absence of a previous cancer were also examined (Table 1). After variants present in any of the sporadic angiosarcomas were excluded, only 8 variants present in more than half of the radiation-associated angiosarcomas remained (Table 4). However, 2 of these variants, a frame-shift deletion in ARID1A (c.3978_3979delGC / p.Gln1327fs*10) and a missense variant in FANCA (c.1127A > T / p.Gln376Leu), were associated with the "role of BRCA1 in DNA damage response" pathway. Furthermore, there were 9 variants that were present in all 16 patient samples (13 radiationassociated angiosarcomas plus 3 sporadic angiosarcomas). Remarkably none of these were associated with the BRCA1 signaling pathway.

\section{Mutational signature analyses}

In the NMF portion of the study, the goal was to identify a mutational signature consisting of a trinucleotide pattern where variants occur rather than identify specific genes with variation. We utilize two human angiosarcoma datasets: (1) the samples in the current Beaumont breast cancer angiosarcoma dataset with 10 or more mutations and (2) the previously published WTSI dataset with mutation data for 11 angiosarcomas that developed in various anatomical locations [7]. These two datasets, with the data normalized according to equal trinucleotide frequencies, are referred to hereafter as the Bang dataset 
Table 2: Variants found in more than two-thirds ( $\geq 9$ of 13) of the radiation-induced angiosarcoma cases and are potentially deleterious (pathogenic or likely pathogenic by ACMG guidelines, listed in HGMD/ClinVar, or predicted to be deleterious by having a CADD score > 20)

\begin{tabular}{lcccccccc}
\hline Chr & Position & $\begin{array}{c}\text { Gene } \\
\text { Symbol }\end{array}$ & $\begin{array}{c}\text { Ref. } \\
\text { Allele }\end{array}$ & $\begin{array}{c}\text { Alt. } \\
\text { Allele }\end{array}$ & Protein Variant & $\begin{array}{c}\text { Cases With } \\
\text { Variant }\end{array}$ & Impact & Classification \\
\hline 1 & $27,100,182$ & ARID1A & GC & - & p.Q1327fs"10 & 9 & FS & Likely Pathogenic \\
1 & $193,111,246$ & CDC73 & AG & - & - & 11 & NC & VUS \\
2 & $47,635,536$ & MSH2 & T & - & - & 10 & NC & VUS \\
2 & $48,032,881$ & MSH6 & ATCT & - & - & 11 & NC & VUS \\
5 & $56,180,645$ & MAP3K1 & G & T & p.W1325L & 12 & MS & VUS \\
5 & $170,827,869$ & NPM1 & T & A & p.N203K & 12 & MS & VUS \\
7 & $55,228,029$ & EGFR & G & A & p.C499Y & 13 & MS & VUS \\
7 & $140,449,164$ & BRAF & C & T & p.V639I & 13 & MS & VUS \\
7 & $140,449,185$ & BRAF & G & A & p.P632S & 13 & MS & VUS \\
13 & $32,907,546$ & BRCA2 & T & - & - & 12 & NC & VUS \\
15 & $40,501,853$ & BUB1B & C & T & p.P721S & 11 & MS & VUS \\
17 & $29,545,994$ & NF1 & - & T & - & 12 & NC & VUS \\
\hline
\end{tabular}

Impact: frameshift (FS), missense (MS), non-coding (NC). ACMG Classification: unknown significance (VUS).

(B for Beaumont and ang for angiosarcoma) and the WTSIang dataset, respectively. Two human datasets from UCSF were also included. One contained three samples from two patients who developed IR-induced sarcomas (the P12 dataset). The other is for two samples from a UV-associated squamous cell carcinoma of the scalp (the P3 dataset). The trinucleotide mutational signature for UV was previously defined; thus, inclusion of the P3

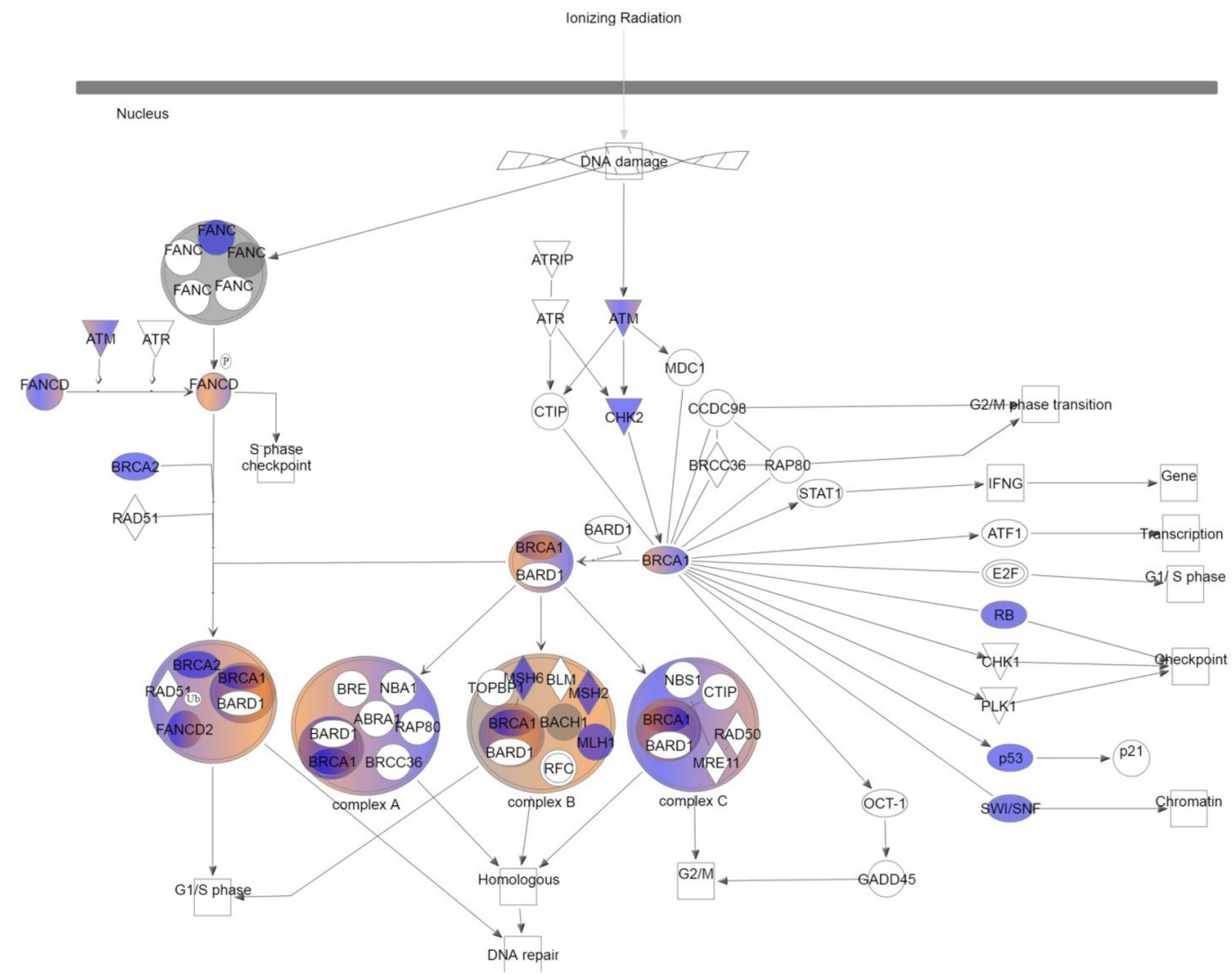

Figure 1: Role of BRCA1 in DNA damage response. Color indicates variants were present in at least 1 case of radiation-induced angiosarcoma after filtering common variants. Blue: loss of function; red: gain of function; grey: inferred normal. 
Table 3: Variants found in both the primary radiation-induced angiosarcoma as well as the matched local recurrence $(n=2$ patients)

\begin{tabular}{lccccccc}
\hline Chr & Position & $\begin{array}{c}\text { Gene } \\
\text { Symbol }\end{array}$ & $\begin{array}{c}\text { Ref. } \\
\text { Allele }\end{array}$ & Alt. Allele & Protein Variant & Impact & Classification \\
\hline 1 & $11,293,378$ & MTOR & A & - & - & NC & VUS \\
1 & $120,510,722$ & NOTCH2 & T & C & p-E414E & syn & Likely Benign \\
2 & $48,032,881$ & MSH6 & ATCT & - & - & NC & VUS \\
5 & $170,818,300$ & NPM1 & T & - & - & NC & VUS \\
5 & $170,827,869$ & NPM1 & T & A & p-N203K & MS & VUS \\
11 & $108,188,279$ & ATM & T & - & - & NC & VUS \\
11 & $108,196,725$ & ATM & AAT & - & - & NC & VUS \\
13 & $32,907,546$ & BRCA2 & T & - & - & NC & VUS \\
15 & $40,501,853$ & BUB1B & C & T & p-P721S & MS & VUS \\
16 & $2,138,213$ & TSC2 & T & A & - & SSL & VUS \\
17 & $41,249,370$ & BRCA1 & A & - & - & NC & VUS \\
19 & $4,110,576$ & MAP2K2 & C & A & p-S127S & syn & VUS \\
22 & $41,527,414$ & EP300 & T & G & p-V435V & syn & Likely Benign \\
22 & $41,565,478$ & EP300 & T & - & - & NC & VUS \\
\hline
\end{tabular}

Impact: missense (MS), synonymous (syn), splice site loss (SSL), non-coding (NC). ACMG Classification: unknown significance (VUS).

data serves as a positive control for a non-IR-associated malignancy. The combined $\mathrm{P} 12$ and $\mathrm{P} 3$ datasets are referred to as the P123 dataset (denoted UCSF in Figure $2 \mathrm{~A} / 2 \mathrm{~B})$.

Pooling of these datasets results in three stably extracted trinucleotide signatures (Supplementary Figure 1; Figure 2). The 3 signatures extracted from the WTSIang + P123 + Bang dataset are shown in Figure $2 \mathrm{C}$, for which the exposure graph is shown in Figure $2 \mathrm{~A}$ and $2 \mathrm{~B}$. These signatures are not highly correlated with each other (Supplementary Table 5, rows 11-13, cols. I-K), suggesting that they may represent separate mutational processes. One of the three NMF signatures from the full dataset is highly correlated (0.999) with the previously defined UV signature [9], which reflects the inclusion of the P3 data in the full dataset and simply demonstrates that this previously defined signature can still be extracted with the other datasets included along with P3 in the present analysis. Of greater substantive interest, the second of the three signatures extracted from the full dataset has a correlation of 0.790 with the previously defined IRa signature present in known IR-induced human cancers $[9,12]$. We see moreover from row 15, col. E of Supplementary Table 5 that even when only the Bang dataset is utilized, the second of the three signatures that can be extracted (albeit with poorer stability properties than for the three signatures extracted from the full dataset) is also correlated 0.706 with the previously defined IRa signature. Thus, whereas also including the WTSIang and the P1-2 and P3 datasets improves the stability of the extracted NMF signatures, the noted correlation with the previously defined IRa signature does not depend on that augmentation of the dataset utilized for NMF.

Figure 2A shows the NMF exposures for the extraction of three signatures from the full dataset. It is clear from this figure that there are far less mutations in the Bang than in the other datasets, and that the $\mathrm{P} 3$ skin cancer samples have by far the most mutations. One important property to note for the form of NMF being utilized is that the signatures extracted are not directly affected by even very large differences among the samples in the number of mutations per sample because the input frequencies are converted to relative frequencies as the first step in the NMF iterative process [13].

In Figure 2B, the exposure results have been converted to a proportional representation for each of the signatures. An observation made by Davidson et al. [9] that applies here as well is that all of the extracted signatures are present in almost all of the cancer samples, but the proportionate representations differ greatly for the UV-associated (Signature 1) and the IR-associated samples. Most importantly in terms of questions of interest involving angiosarcomas, the dark blue representing the signature that is highly correlated with the previously defined IRa signature is present in almost all of the Bang samples in similar proportions to its representation in the WTSIang and also the P1-2 dataset samples. Figure 2C displays each of the three signatures. All three signatures are characterized by predominantly $\mathrm{C} \rightarrow \mathrm{T}$ substitutions but the weights differ markedly by neighboring nucleotides. 


\section{DISCUSSION}

Investigation of radiation-associated angiosarcomas has always proven difficult due to the extremely low incidence [14]. This means that case studies are the predominant type of investigation found in the literature. Despite the low number of patients in each study, there has been significant agreement among them. The interval between primary breast carcinoma and eventual radiationassociated angiosarcoma generally has a median of
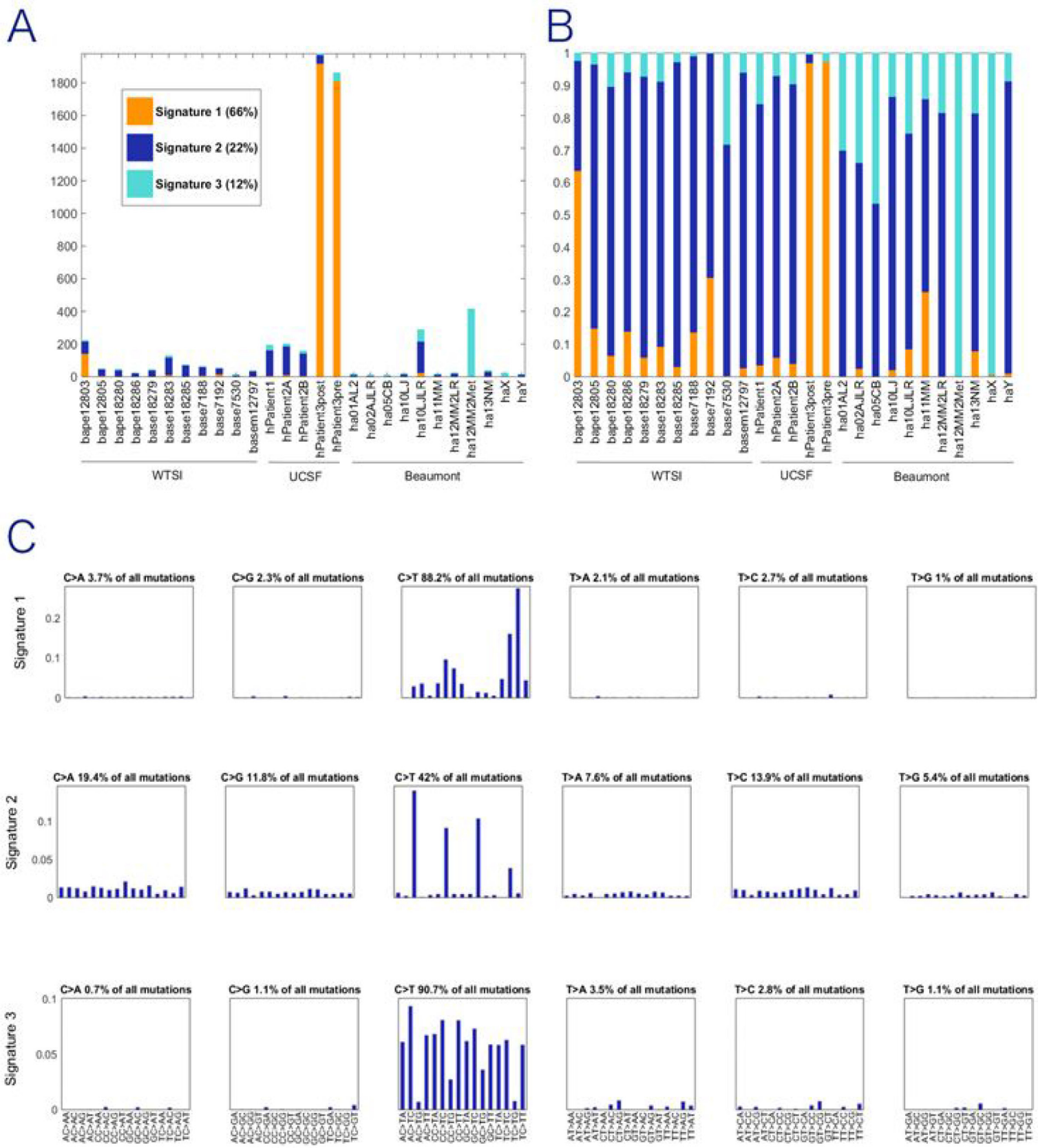

Figure 2: Exposures for NMF trinucleotide mutational signatures for combined dataset. WTSI represents the angiosarcomas reported in Behjati et al. (7); UCSF represents the irradiated samples reported in Davidson et al. (9); Beaumont represents the new data first reported in this paper. (A) Count of mutations in each tissue sample attributed to each signature. (B) Proportion of mutations in each tissue sample attributed to each signature. (C) Three discrete mutational signatures were identified the pooled analysis. The plots show the distribution of the six mutation types defined by the pyrimidine base in each signature, as inferred from the NMF procedure. Each subgraph within a signature represents one substitution (e.g., $\mathrm{C} \rightarrow \mathrm{A}$ when $\mathrm{C}$ in the reference genome is mutated to $\mathrm{A}$ in the sample). The bars within each sub-graph include the nucleotides in the reference genome on either side of the mutation location (e.g., AC > AG represents A at 5', C in the reference mutated to A, and $\mathrm{G}$ at 3'), 96 substitution types shown. All three signatures are characterized by predominantly $\mathrm{C} \rightarrow \mathrm{T}$ substitutions but the weights differ markedly by neighboring nucleotides. Signature 1 is defined by the skin cancer exomes and is the recovered UV signature. 
Table 4: Variants found in more than half of the radiation-induced angiosarcoma cases and 0 of 3 sporadic angiosarcomas

\begin{tabular}{lcccccccc}
\hline Chr & Position & $\begin{array}{c}\text { Gene } \\
\text { Symbol }\end{array}$ & $\begin{array}{c}\text { Ref. } \\
\text { Allele }\end{array}$ & $\begin{array}{c}\text { Alt. } \\
\text { Allele }\end{array}$ & $\begin{array}{c}\text { Protein } \\
\text { Variant }\end{array}$ & $\begin{array}{c}\text { Cases } \\
\text { With } \\
\text { Variant }\end{array}$ & Impact & Classification \\
\hline 1 & $27,100,182$ & ARID1A & GC & - & p.Q1327fs"10 & 9 & FS & Likely Pathogenic \\
2 & $47,600,591$ & EPCAM & T & - & - & 9 & NC & VUS \\
7 & $6,026,541$ & PMS2 & C & T & p.D513N & 7 & MS & VUS \\
11 & $119,156,068$ & CBL & T & A & p.L578Q & 7 & MS & VUS \\
14 & $23,607,210$ & SLC7A8 & T & A & p.G312G & 7 & syn & VUS \\
16 & $89,858,433$ & FANCA & T & A & p.Q376L & 7 & MS & VUS \\
17 & $37,657,470$ & CDK12 & T & - & - & 7 & NC & VUS \\
22 & $41,547,890$ & EP300 & T & A & p.T957T & 9 & syn & VUS \\
\hline
\end{tabular}

Impact: frameshift (FS), missense (MS), synonymous (syn), non-coding (NC). ACMG Classification: unknown significance (VUS).

approximately 7 years with a range between 3 and 25 years [14-19]. This corresponds well with our current cohort that ranged between 3.9 and 13.5 years with a mean of 6.8 years. Additionally disease-free survival was found to be $35 \%$ [14] at 2 years with a median of 16 months [18]. Studies also found high levels of recurrence [17] with local recurrence in up to two thirds of patients and metastasis in more than one thirds [20]. Our cohort did not exhibit this high level of recurrence with only 4 of the 13 cases having recurrences. These poor outcomes are a direct result of the difficulty in diagnosing these angiosarcomas following treatment of the primary breast carcinoma [21]. Additionally, while no study currently demonstrates it, some hypothesize that the incidence of radiation-associated angiosarcoma may increase due to the higher usage of radiation in breast conserving techniques in the treatment of breast cancer.

The current study aimed to discover somatic genomic variants common in radiation-induced angiosarcoma; moreover, given the limited data available, signaling pathways were investigated for high levels of variation which may prove important to guide targeted treatments. In the current study we examined a panel of 160 cancer-related genes. In the radiation-associated angiosarcomas, 44 variants were present in at least two thirds of the 13 cases. In order to identify variants that were more likely to be deleterious, these were further filtered based upon ACMG, HGMD, ClinVar, or CADD. This identified 12 variants, including 6 missense variants, 5 small deletions, and 1 single base insertion. Of interest was the missense variant in EGFR that resulted in an amino acid change from cysteine to tyrosine. It was found in all 13 cases of radiation-associated angiosarcoma. This variant is affects the extracellular ligand-binding domain of EGFR and is predicted to affect the function of the protein by both SIFT and PolyPhen-2 function prediction algorithms. The importance of EGFR was suggested in a study of a cell line derived from a radiation-associated angiosarcoma where inhibition of the VEGFR2/EGFR/ RET axis resulted in decreased cell proliferation [22]. The only other gene with variants in all 13 cases was BRAF, which had 2 separate variants (chr7:140,449,164 and chr7:140,449,185) present in all cases. Murali et al. also found variation in BRAF including single nucleotide variants and amplification in a study of primary angiosarcomas [23].

In order to identify signaling that is highly altered in the radiation-induced angiosarcomas, we focused on variants that were shown to have a damaging effect on gene function without requiring two thirds of the cases to have a given variant. This identified genes involved in the DNA damage response to be highly altered in the all cases of radiation-associated angiosarcoma. Each case had an average of 6 , and as many as 12 , different genes that had variants in the BRCA1 DNA damage response pathway. Previously published case reports have shown that carriers of BRCA1 and BRCA2 mutations formed angiosarcomas following radiation treatment of breast cancer [24-26]. While these case studies did not prove causation, all three recommended consideration of BRCA status in treating primary breast carcinoma. In another study, 3 of 7 cases of radiation-induced angiosarcoma contained BRCA1 or BRCA2 mutations [27]. This group, however, concluded that BRCA status should not be considered in treatment decisions. While the percent of cases with a mutation was high, the incidence among BRCA mutation carriers was low and therefore not worth considering in treatment of the primary carcinoma. Our results may indicate the importance of the BRCA damage response outside of germline BRCA1 and BRCA2 variation. With the high number of genes affected within the tumor genome, the DNA response pathway may prove to be a promising target to combat the high rate of recurrence in these patients. 
Another approach that we explored was that these tumors would have a mutational signature that is characteristic of radiation exposure. Prior work has demonstrated that non-negative matrix factorization is an analytic approach that identifies trinucleotide-based mutational signatures in tumors. This approach is capable of differentiating different mutagenic exposures, including ionizing radiation (IR) [28]. Sherborne et al. used this method to identify 3 mutational signatures that were characteristic of the radiation-induced tumors in a mouse model [12]. These signatures were comprised of specific patterns of tri-nucleotide variation that were not dependent upon the genetic background or the mutational load of a sample. The Beaumont angiosarcoma data alone is too limited to extract NMF signatures that can be viewed with any degree of confidence, even if the usual stability and reconstruction metrics are acceptable. To address this limitation we pooled the Beaumont angiosarcoma data with angiosarcoma data and data for known IR-induced cancers from other published studies [7-9]. The fact that this pooled full dataset analysis also yields a trinucleotide signature that is highly correlated with a previously defined IR-associated signature [9] is of interest, though this result must be viewed as tentative until confirmed based on further data for this rare cancer.

One limitation of the current study is the lack of availability of germline DNA for analysis. This hindered our ability to differentiate between somatic and germline variation. There are several variants that were identified that are often found in familial predisposition syndromes. The BRCA2 (c.1909+22delT) variant was found in 12 of the 13 radiation-associated angiosarcomas in the current study. This same variant was identified as a germline variant leading to susceptibility to breast/ovarian cancer (ClinVar: RCV000119249.1) but has recently been reannotated as benign (ClinVar:RCV000043921.6). Several others genes with potential germline variants were found when examining the "role of BRCA1 in DNA Damage Response" pathway. This included variants in ATM, BRCA1, CHEK2, FANCA, and FANCD2 that are annotated in the ClinVar database. The majority of these variants occurred in only 1 patient within our cohort. The only exception is the variant in FANCD2 (c.2613A > C / p.K871N) which is in ClinVar associated with Fanconi anemia. In addition, our study was limited by the scope of our sequencing panel. While able to detect variation in 160 genes, the panel did not allow the detection of amplification; in particular, we were unable to detect $M Y C$ amplification. Amplification of MYC has been shown to be an important marker of radiation-associated angiosarcoma while it is not common in sporadic angiosarcomas [29-31]. That said, MYC showed variants in 5 patients with other patients showing variants in genes associated with MYC mediated apoptosis signaling.

Another notable limitation is the absence of validation of the discovered variants by Sanger sequencing in order to eliminate false positives due to DNA isolation or sequencing artifacts. Unfortunately our ability for subsequent validation was eliminated by two factors in this study. First the use of laser capture microdissection greatly reduced the amount of DNA isolated from our current samples. While LCM has the benefit of focusing analysis specifically on tumor cells, the isolated DNA is only sufficient for a single sequencing run. The second important factor is the extreme rarity of this disease. As with many studies into radiation-associated angiosarcoma, we were limited by the number of available samples for study. Given the low incidence, it was difficult to compile a large cohort of available patient samples despite going back as far as 20+ years. This proved even more difficult when attempting to obtain matching recurrence and metastatic biospecimens. Ideally this study will be complemented by a future, multi-institution study with the intention of confirming and expanding this study. In order to combat these limitations, we tried to focus on variants that had evidence that indicated their potential deleterious nature. Focusing on variants categorized as pathogenic or likely pathogenic based upon ACMG guidelines, listed in HGMD/ClinVar, or having a CADD (Combined Annotation Dependent Depletion) score greater than 20 hopefully emphasized potential variants that are likely to be true positives that result in damaged phenotype.

Also of note was the absence of variants in TP53 in the results of this study. In an earlier study of second malignant neoplasms in pediatric patients [8], identifying germline TP53 variants was shown to be important in identifying patients at high risk for development of secondary malignancy. Of the 13 cases of radiationassociated angiosarcoma, only 4 contained variants in TP53. This included a case with 2 variants confirmed as somatic and pathogenic by COSMIC (COSM3388195 and COSM3378350). Interestingly, none of the 3 cases of sporadic angiosarcoma contained a TP53 variant. This suggests a role for TP53 in a subset of radiation-induced angiosarcomas and supports the conclusion of Sherborne et al that identifying germline TP53 variants prior to radiation therapy may be beneficial for treatment selection and post-treatment monitoring. However, additional studies with germline TP53 sequencing would need to be completed on this adult population of patients with radiation-associated secondary malignancies.

Studies of radiation-induced angiosarcoma are generally limited by the low incidence, and it is important to conduct multi-center investigations to expand on the currently available studies. That being said, the current study identified individual genomic variants including variants in EGFR and BRAF that occurred in all samples of this study. In addition, genes associated with the role of BRCA1 in DNA damage response are commonly altered in all of the current cases of radiation-induced angiosarcoma. This might suggest that radiation-induced angiosarcomas are defective in the homologous recombination repair 
pathway which might render them sensitive to platinumbased chemotherapy and poly ADP ribose polymerase (PARP) inhibitors. Further investigation with either whole genome or exome sequencing is warranted. This would enable the discovery and confirmation of potential drug targets but also better unmask a potential radiationassociated signature.

\section{MATERIALS AND METHODS}

\section{Human research protection}

The Beaumont Human Investigation Committee approved all work under an approved research protocol (IRB \#2014-083).

\section{Patient selection and laser capture microdissection (LCM)}

Angiosarcoma cases from 2005 through 2015 were identified using databases available through the Department of Radiation Oncology. Inclusion criteria included previous breast cancer diagnosis and site of primary angiosarcoma to be in the ipsilateral breast as the earlier cancer. The majority of these patients received whole breast irradiation; therefore, secondary malignancies were presumed to be in-field.

Archival formalin-fixed paraffin-embedded (FFPE) tumor samples with the diagnosis of breast angiosarcoma were obtained from Beaumont Health Department of Pathology. Hematoxylin-eosin slides were reviewed by a single pathologist for confirmation of angiosarcoma and identification of tumor location. FFPE sections were cut at $5 \mu \mathrm{m}$ thickness and mounted onto polyethylene naphthalate membrane glass slides (2 sections per slide). Before deparaffinization, slides were placed into an oven set at $60^{\circ} \mathrm{C}$ for 15 minutes then stained with hematoxylin and dehydrated through a series of graded ethanol and xylene steps. Pathologist-identified areas were microdissected with both UV and IR lasers using the ArcturusXT Laser Capture Microdissection system (Applied Biosystems, Carlsbad, CA) onto CapSure HS LCM Caps (Applied Biosystems, Carlsbad, CA). Four caps were filled per sample from the dissection of 1 to 4 sections.

\section{DNA isolation}

DNA was isolated from LCM caps using GeneRead DNA FFPE Tissue Kit (Qiagen, Valencia, CA) according to manufacturer's protocol. However, the deparaffinization step was omitted starting with the proteinase $\mathrm{K}$ digestion step followed by incubation at $56^{\circ} \mathrm{C}$ for $16 \mathrm{hr}$. Quality and amplifiable DNA material was assessed with the GeneRead DNA QuantiMIZE Kit (Qiagen, Valencia, CA).

\section{Library preparation}

Targeted enrichment multiplex PCR of 160 genes was done using GeneRead DNAseq Comprehensive Cancer Panel V2 in combination with GeneRead DNAseq Panel PCR Kit V2 (Qiagen, Valencia, CA) following manufacturer's protocol. Starting amount of amplifiable DNA and number of PCR cycles for initial library amplification were determined with GeneRead DNA QuantiMIZE kit. Sample purification was done using Agencourt AMPure XP Beads (Beckman Coulter, Brea, CA). Library construction was performed using GeneRead Illumina based DNA Library Prep Kits with sample multiplexing done using GeneRead Adapter I Set 12-plex (Qiagen, Valencia, CA). The resulting barcoded libraries were quantified using GeneRead DNAseq Quantification Kit, then pooled together before being submitted to a NextSeq 500 sequencer (Illumina, San Diego, CA) using a $2 \times 150$ paired-end sequencing strategy.

\section{Alignment}

Primary basecall sequencing outputs were converted to FASTQ format and demultiplexed on Illumina's BaseSpace. Read trimming and alignment were performed with NextGENe software (SoftGenetics, State College, PA). Reads with median base Phred33 quality score $\geq 10$, $\leq 3$ uncalled bases, $\geq 20$ total bases called, and with $\leq 3$ bases of quality $\leq 10$ were retained for further analysis. Alignment of the sequencing reads to the human reference genome (v37.3, dbSNP 135) was performed with a NextGENe proprietary algorithm.

\section{Mutation analysis}

Somatic mutations were called with NextGENe program on tumor only based upon mutation percentage $\geq 5 \%$ and total read coverage $\geq 250$. The coverage requirement is ignored for mutations that are homozygous. If the mutation occurs with a mutation percentage $\leq$ $80 \%$, the mutation is excluded if the ratio of forward and reverse reads is $<0.1$. Variants were then annotated using Ingenuity Variant Analysis (IVA, 2017 Winter Release) software (Qiagen, Redwood City, CA). Variants referenced in IVA proprietary Knowledge Base with an allele frequency $\geq 1 \%$ in the general population (using the 1000 Genomes Project, the National Heart, Lung, and Blood Institute Exome Sequencing Project, the Allele Frequency Community, and the Exome Aggregation Consortium) were considered SNPs and were discarded.

\section{Trinucleotide-based mutational signatures}

Radiation-specific alterations of genomic sequences have first been studied by applying a non-negative factorization (NMF) approach $[12,13,28]$ to the filtered somatic mutations identified in our samples. 
In order to extract stable signatures, the limited set of mutations detected in the angiosarcoma samples sequenced here has been complemented with previously published angiosarcoma mutation data [7] as well as with human tumor single nucleotide variants identified in malignancies known to be induced by radiation (ionizing, IR, or ultraviolet, UV) (Supplementary Table 1). The counts of the mutation motifs have then been normalized according to equal trinucleotide frequencies (following the second normalization approach used by $[9,32]$. It is the second of those two normalization approaches that equalizes the probability of a purely random mutation at any one trinucleotide, which helps to isolate the variations in the data of prime interest in this work.

Thus, following previously published methods [9], we pooled normalized sequencing data for the Beaumont angiosarcoma breast cancer samples containing at least 10 mutations each (hereafter referred to as the "Bang" dataset) with normalized previously published sequencing data for the coding portion of whole genome or whole exome sequencing data for angiosarcomas [7] (the "WTSIang" dataset) as well as with normalized data for two patients whose cancers are known to have been induced by ionizing radiation (IR) [8] (the "P1-2" dataset) plus data for a third patient whose skin cancer was known to be caused by exposure to sunlight (the "P3" dataset). What is referred to below as the full dataset combines the WTSIang, the P1-2, the P3, and the Bang component datasets.

Non-negative matrix factorization (NMF) was performed following protocols developed by WTSI [13]. Signature stability and reconstruction error quality metrics were computed to estimate the number of signatures supported by the numbers of single nucleotide variants.

\section{Author contributions}

Bryan J. Thibodeau (experimental design, data analysis and interpretation, manuscript preparation); Vincent Lavergne (data analysis and interpretation, manuscript preparation); Nayana Dekhne (study conception and design); Pamela Benitez (study conception and design); Mitual Amin (study conception and design); Samreen Ahmed (data collection, laboratory experiments); Jean L. Nakamura (data analysis and interpretation, manuscript preparation); Philip R. Davidson (data analysis); Alice O. Nakamura (data analysis); Inga S. Grills (patient identification); Peter Y. Chen (patient identification); Jessica Wobb (experimental design, data collection); George D. Wilson (study conception and design, data interpretation, manuscript preparation).

\section{ACKNOWLEDGMENTS AND FUNDING}

This work was supported by a generous philanthropic gift from the Barbara Miller Fund.

\section{CONFLICTS OF INTEREST}

The authors declare no potential conflicts of interest.

\section{REFERENCES}

1. Adem C, Reynolds $\mathrm{C}$, Ingle $\mathrm{JN}$, Nascimento AG. Primary breast sarcoma: clinicopathologic series from the Mayo Clinic and review of the literature. Br J Cancer. 2004; 91:237-41. https://doi.org/10.1038/sj.bjc.6601920.

2. Kirova YM, Vilcoq JR, Asselain B, Sastre-Garau X, Fourquet A. Radiation-induced sarcomas after radiotherapy for breast carcinoma: a large-scale single-institution review. Cancer. 2005; 104:856-63. https://doi.org/10.1002/ cncr. 21223.

3. Sheppard DG, Libshitz HI. Post-radiation sarcomas: a review of the clinical and imaging features in 63 cases. Clin Radiol. 2001; 56:22-9. https://doi.org/10.1053/ crad.2000.0599.

4. Mentzel T, Schildhaus HU, Palmedo G, Buttner R, Kutzner H. Postradiation cutaneous angiosarcoma after treatment of breast carcinoma is characterized by MYC amplification in contrast to atypical vascular lesions after radiotherapy and control cases: clinicopathological, immunohistochemical and molecular analysis of 66 cases. Mod Pathol. 2012; 25:75-85. https://doi.org/10.1038/ modpathol.2011.134.

5. Guo T, Zhang L, Chang NE, Singer S, Maki RG, Antonescu CR. Consistent MYC and FLT4 gene amplification in radiation-induced angiosarcoma but not in other radiationassociated atypical vascular lesions. Genes Chromosomes Cancer. 2011; 50:25-33. https://doi.org/10.1002/gcc.20827.

6. Hadj-Hamou NS, Lae M, Almeida A, de la Grange P, Kirova Y, Sastre-Garau X, Malfoy B. A transcriptome signature of endothelial lymphatic cells coexists with the chronic oxidative stress signature in radiation-induced postradiotherapy breast angiosarcomas. Carcinogenesis. 2012; 33:1399-405. https://doi.org/10.1093/carcin/bgs 155.

7. Behjati S, Tarpey PS, Sheldon H, Martincorena I, Van Loo P, Gundem G, Wedge DC, Ramakrishna M, Cooke SL, Pillay N, Vollan HKM, Papaemmanuil E, Koss H, et al. Recurrent PTPRB and PLCG1 mutations in angiosarcoma. Nat Genet. 2014; 46:376-9. https://doi.org/10.1038/ng.2921.

8. Sherborne AL, Lavergne V, Yu K, Lee L, Davidson PR, Mazor T, Smirnoff IV, Horvai AE, Loh M, DuBois SG, Goldsby RE, Neglia JP, Hammond S, et al. Somatic and Germline TP53 Alterations in Second Malignant Neoplasms from Pediatric Cancer Survivors. Clin Cancer Res. 2017; 23:1852-61. https://doi.org/10.1158/1078-0432.CCR-160610 .

9. Davidson PR, Sherborne AL, Taylor BS, Nakamura AO, Nakamura JL. A pooled mutational analysis identifies ionizing radiation-associated mutational signatures conserved between mouse and human malignancies. Sci Rep. 2017; 7:7645. https://doi.org/10.1038/s41598-017-07888-0. 
10. Kumar P, Henikoff S, Ng PC. Predicting the effects of coding non-synonymous variants on protein function using the SIFT algorithm. Nat Protoc. 2009; 4:1073-81. https:// doi.org/10.1038/nprot.2009.86.

11. Adzhubei IA, Schmidt S, Peshkin L, Ramensky VE, Gerasimova A, Bork P, Kondrashov AS, Sunyaev SR. A method and server for predicting damaging missense mutations. Nat Methods. 2010; 7:248-9. https://doi. org/10.1038/nmeth0410-248.

12. Sherborne AL, Davidson PR, Yu K, Nakamura AO, Rashid M, Nakamura JL. Mutational Analysis of Ionizing Radiation Induced Neoplasms. Cell Reports. 2015; 12:1915-26. https://doi.org/10.1016/j.celrep.2015.08.015.

13. Alexandrov LB, Nik-Zainal S, Wedge DC, Campbell PJ, Stratton MR. Deciphering signatures of mutational processes operative in human cancer. Cell Rep. 2013; 3:246-59. https://doi.org/10.1016/j.celrep.2012.12.008.

14. Strobbe LJ, Peterse HL, van Tinteren H, Wijnmaalen A, Rutgers EJ. Angiosarcoma of the breast after conservation therapy for invasive cancer, the incidence and outcome. An unforseen sequela. Breast Cancer Res Treat. 1998; 47:101-9. https://doi.org/10.1023/A:1005997017102.

15. Shah S, Rosa M. Radiation-Associated Angiosarcoma of the Breast: Clinical and Pathologic Features. Arch Pathol Lab Med. 2016; 140:477-81. https://doi.org/10.5858/arpa.20140581-RS.

16. Uryvaev A, Moskovitz M, Abdach-Bortnyak R, Hershkovitz D, Fried G. Post-irradiation angiosarcoma of the breast: clinical presentation and outcome in a series of six cases. Breast Cancer Res Treat. 2015; 153:3-8. https://doi. org/10.1007/s10549-015-3506-0.

17. Torres KE, Ravi V, Kin K, Yi M, Guadagnolo BA, May CD, Arun BK, Hunt KK, Lam R, Lahat G, Hoffman A, Cormier JN, Feig BW, et al. Long-term outcomes in patients with radiationassociated angiosarcomas of the breast following surgery and radiotherapy for breast cancer. Ann Surg Oncol. 2013; 20:1267-74. https://doi.org/10.1245/s10434-012-2755-y.

18. Seinen JM, Styring E, Verstappen V, Vult von Steyern F, Rydholm A, Suurmeijer AJ, Hoekstra HJ. Radiationassociated angiosarcoma after breast cancer: high recurrence rate and poor survival despite surgical treatment with R0 resection. Ann Surg Oncol. 2012; 19:2700-6. https://doi.org/10.1245/s10434-012-2310-x.

19. D'Angelo SP, Antonescu CR, Kuk D, Qin L, Moraco N, Carvajal RC, Chi P, Dickson MA, Gounder M, Keohan ML, Singer S, Schwartz GK, Tap WD. High-risk features in radiation-associated breast angiosarcomas. Br J Cancer. 2013; 109:2340-6. https://doi.org/10.1038/bjc.2013.590.

20. Depla AL, Scharloo-Karels CH, de Jong MA, Oldenborg S, Kolff MW, Oei SB, van Coevorden F, van Rhoon GC, Baartman EA, Scholten RJ, Crezee J, van Tienhoven G. Treatment and prognostic factors of radiation-associated angiosarcoma (RAAS) after primary breast cancer: a systematic review. Eur J Cancer. 2014; 50:1779-88. https:// doi.org/10.1016/j.ejca.2014.03.002.
21. Daniels BH, Ko JS, Rowe JJ, Downs-Kelly E, Billings SD. Radiation-associated angiosarcoma in the setting of breast cancer mimicking radiation dermatitis: A diagnostic pitfall. J Cutan Pathol. 2017; 44:456-61. https://doi.org/10.1111/ cup.12917.

22. Azzariti A, Porcelli L, Mangia A, Saponaro C, Quatrale AE, Popescu OS, Strippoli S, Simone G, Paradiso A, Guida M. Irradiation-induced angiosarcoma and anti-angiogenic therapy: a therapeutic hope? Exp Cell Res. 2014; 321:2407. https://doi.org/10.1016/j.yexcr.2013.12.018.

23. Murali R, Chandramohan R, Moller I, Scholz SL, Berger M, Huberman K, Viale A, Pirun M, Socci ND, Bouvier N, Bauer S, Artl M, Schilling B, et al. Targeted massively parallel sequencing of angiosarcomas reveals frequent activation of the mitogen activated protein kinase pathway. Oncotarget. 2015; 6:36041-52. https://doi.org/10.18632/ oncotarget.5936.

24. West JG, Weitzel JN, Tao ML, Carpenter M, West JE, Fanning C. BRCA mutations and the risk of angiosarcoma after breast cancer treatment. Clin Breast Cancer. 2008; 8:533-7. https://doi.org/10.3816/CBC.2008.n.066.

25. Williams SB, Reed M. Cutaneous angiosarcoma after breast conserving treatment for bilateral breast cancers in a BRCA-1 gene mutation carrier--a case report and review of the literature. Surgeon. 2009; 7:250. https://doi.org/10.1016/ S1479-666X(09)80094-2.

26. Parvez E, Popovic S, Elavathil L, Okawara G, Hodgson N. Early Occurrence of Angiosarcoma in a Woman With a BRCA2 Gene Variation of Unknown Significance Treated With Breast-Conserving Therapy for Bilateral Ductal Carcinoma: A Case Report. Clin Breast Cancer. 2015; 15:536-8. https://doi.org/10.1016/j.clbc.2015.06.011.

27. Kadouri L, Sagi M, Goldberg Y, Lerer I, Hamburger T, Peretz T. Genetic predisposition to radiation induced sarcoma: possible role for BRCA and p53 mutations. Breast Cancer Res Treat. 2013; 140:207-11. https://doi. org/10.1007/s10549-013-2621-z.

28. Alexandrov LB, Stratton MR. Mutational signatures: the patterns of somatic mutations hidden in cancer genomes. Curr Opin Genet Dev. 2014; 24:52-60. https://doi. org/10.1016/j.gde.2013.11.014.

29. Wade MA, May FE, Onel K, Allan JM. Does radiationinduced c-MYC amplification initiate breast oncogenesis? Mol Cell Oncol. 2015; 3:e1010950. https://doi.org/10.1080 /23723556.2015.1010950.

30. Ginter PS, Mosquera JM, MacDonald TY, D'Alfonso TM, Rubin MA, Shin SJ. Diagnostic utility of MYC amplification and anti-MYC immunohistochemistry in atypical vascular lesions, primary or radiation-induced mammary angiosarcomas, and primary angiosarcomas of other sites. Hum Pathol. 2014; 45:709-16. https://doi. org/10.1016/j.humpath.2013.11.002.

31. Huang SC, Zhang L, Sung YS, Chen CL, Kao YC, Agaram NP, Singer S, Tap WD, D'Angelo S, Antonescu CR. Recurrent CIC Gene Abnormalities in Angiosarcomas: 
A Molecular Study of 120 Cases With Concurrent Investigation of PLCG1, KDR, MYC, and FLT4 Gene Alterations. Am J Surg Pathol. 2016; 40:645-55. https:// doi.org/10.1097/PAS.0000000000000582.

32. Alexandrov LB, Nik-Zainal S, Wedge DC, Aparicio SA, Behjati S, Biankin AV, Bignell GR, Bolli N, Borg A,
Borresen-Dale AL, Boyault S, Burkhardt B, Butler AP, et al, and Australian Pancreatic Cancer Genome Initiative, and ICGC Breast Cancer Consortium, and ICGC MMML-Seq Consortium, and ICGC PedBrain. Signatures of mutational processes in human cancer. Nature. 2013; 500:415-21. https://doi.org/10.1038/nature12477. 